\title{
Structural Factors Influencing the Volatile Sensitivity of Polymer Coated Piezoelectric Micromechanical Resonators
}

\author{
Ling Sieben-Xu, Devrez M. Karabacak, Daan Wouters, Wout Knoben, Yvonne van Andel, \\ Sywert H. Brongersma, Mercedes Crego Calama \\ Holst Centre/imec the Netherlands, High Tech Campus 31, 5656AE, the Netherlands
}

\begin{abstract}
Polymer-coated piezoelectric microbridge resonators were demonstrated to be highly sensitive towards volatile organic compounds. Here, we present a comprehensive experimental study into several important factors governing the modal sensitivity, namely the modal resonance frequency, piezoelectric transducer size, and functionalization layer thickness. Results indicate the lower frequency length flexural modes yield higher normalized frequency shifts in comparison to higher order width-flexural or torsional modes. The sensitivity of resonator sensors can be further enhanced by reducing the piezoelectric patch size and increasing the thickness of polymer.
\end{abstract}

Key words: electronic nose, volatile organic compound, piezoelectric microbridge, polymer

\begin{abstract}
Introduction
Compact, low power, low cost and integrated sensors are in a growing need to detect low concentration of volatile organic compounds (VOCs), with potential applications ranging from indoor air quality monitoring to industrial leakage detection and health care [1]. However, existing detection approaches, mainly based on analytical approaches of chromatography and spectrometry, have several drawbacks, such as being not easily scalable in size, high power consumption and costly, preventing their onfield usage. Alternatively, use of partially selective receptors like polymers in arrays for detection and identification of volatiles is a promising approach, often referred to as "electronic nose" system. For this task, micromechanical resonators are widely viewed as suitable transducers with intrinsic advantages of low power consumption, CMOS technology compatibility, easily scalable and arrayable.
\end{abstract}

Previously, polymer-coated doubly clamped microbridge resonator sensor has been demonstrated to detect low concentrations of volatile compounds by monitoring their resonance frequency shift [2, 3], among which the resonator integrated with piezoelectric transducer in [2] is particularly attractive due to its ultra low power consumption and more promising integration capabilities. The key factors of microbridge resonator which influence its sensitivity, however, have not been investigated yet. In this paper, we explore several factors of beam resonator integrated with piezoelectric transducer (schematized in Fig. 2(a) inset), such as modal resonance frequency, piezoelectric patch size and polymer coating. The results will enable us to further optimize the sensitivity of the resonator sensor, and therefore offer the insight for their potential in future applications in "electronic nose" systems.

\section{Fabrication and functionalization}

The doubly clamped microbridge resonators were fabricated by a process flow that combines surface and bulk micromachining processes on silicon ( $\mathrm{Si}$ ) substrates. Firstly, a sacrificial $500 \mathrm{~nm}$ thick silicon oxide $\left(\mathrm{SiO}_{2}\right)$ was deposited as a $\mathrm{Si}$ deep reactive ion etching (DRIE) stop layer by low-pressure chemical vapor deposition (LPCVD). Later, the beam layer, a $500 \mathrm{~nm}$ thick low-stress silicon nitride was deposited by low-pressure chemical vapor deposition. For piezoelectric transduction, a patch of was formed by bottom-up fabrication from platinum $(\mathrm{Pt}) /$ aluminum nitride (AIN) / platinum (Pt) stack $(100 \mathrm{~nm} / 400 \mathrm{~nm} / 100$ $\mathrm{nm}$ ). The microbridge was formed by a throughwafer Si DRIE, performed from the backside. Finally, the sacrificial $\mathrm{SiO}_{2}$ stop layer was removed in buffered oxide etchant, completing the release of the structures. An image of a typical beam array is shown in Fig. 1.

For exploring the factors influencing the resonator sensitivity, ethanol is selected as a suitable representative volatile compound, and poly(methyl methacrylate), PMMA, is correspondingly used as the sensing layer due its well known affinity to absorb ethanol $[2,4,5]$. 
This selection, however, does not limit the implications of the results, as similar behavior is expected for all the other polymers with swelling effect, albeit at potentially different levels.

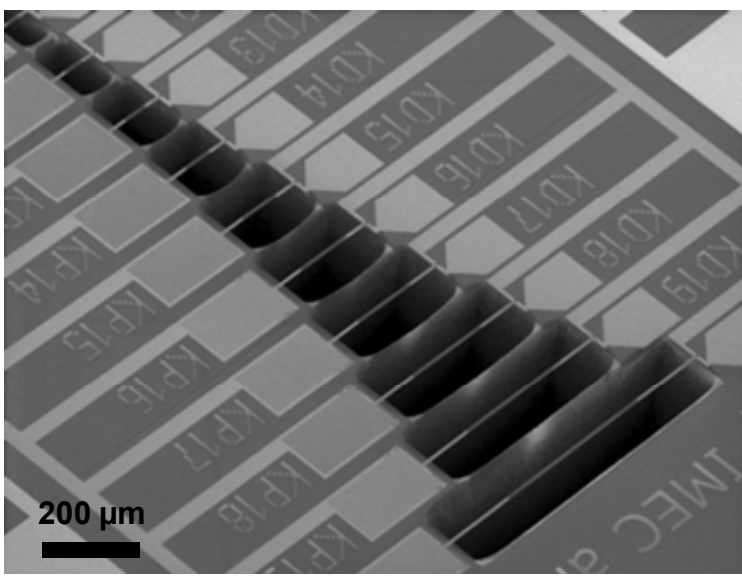

Fig. 1 An example scanning electron microscope image of suspended beam array with $70 \mu \mathrm{m}$ width and lengths ranging from $50-750 \mu \mathrm{m}$, and their piezoelectric patch covering $25 \%$ of beam length.

A dilute printing solution was prepared with PMMA in methyl benzoate, and coated on to the backside of each beam resonator using a commercial inkjet printer with droplets ejected through the deeply etched window, as the nozzle position was swept along the length of the structure. The thickness of the polymer is determined by the concentration of the ink and the number of printed droplets. Since, due to viscosity limits, the concentration of polymer in the ink is limited, thicker polymer layers may be obtained by printing multiple layers on top of each other.

\section{Measurement approach and results}

Microbridge resonator sensors are calibrated in a stainless steel flow chamber with a constant flow rate $(0.2 \mathrm{nl} / \mathrm{min})$ at room temperature. Various volatile concentration levels are obtained by diluting the saturated vapor with dry nitrogen $\left(\mathrm{N}_{2}\right)$.

Each resonator has tens of resonance modes, and they can be recorded by measuring the conductance $(G)$ and susceptance $(B)$ during a frequency sweeping using an impedance analyzer.

Example recorded conductance-frequency (G-f) curve around one of the resonance frequencies $(801 \mathrm{kHz})$ is shown in Fig. 2 for a $750 \mu \mathrm{m}$ long and $70 \mu \mathrm{m}$ wide resonator sensor with $375 \mu \mathrm{m}$ long (50\% of beam length) piezoelectric patch.

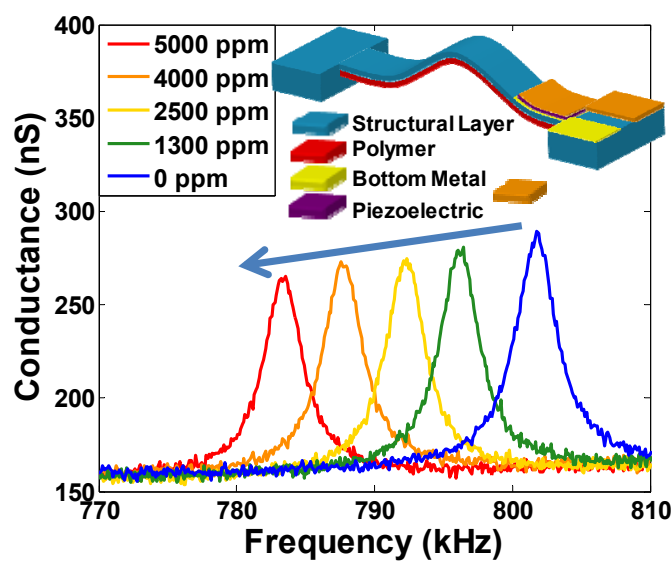

Fig. 2 Modal frequency shift of a PMMA-coated microbridge resonator (length $L=750 \mu \mathrm{m}$, width $=70$ $\mu \mathrm{m}$ and $500 \mathrm{~nm}$ thick silicon nitride, piezoelectric patch coverage 0.5L) upon exposure to increasing concentrations of ethanol. Inset shows the schematic of the microbridge structure in its fundamental mode shape, with the individual structural layers as indicated.

Once the sensor is exposed to ethanol, PMMA coated on backside of beam starts to absorb the volatile, resulting in both mass increase and swelling, and the combined effect of increased inertia and axial stress formation due to swelling results in a negative resonance frequency shift, indicated by the arrow shown in Fig. 2.

\section{Sensitivity of resonance modes}

The modal sensitivity of a resonant sensor, which depends on vibration mode shape, geometry and materials, can be defined as $S_{V}=-$ $\Delta f /\left(f_{n} \cdot C_{v}\right)$, where the relative frequency shift $\Delta f$ per vapor concentration $C_{v}$ is normalized to the modal frequency $f_{n}$, and $n$ is the mode number.

The resonator sensor can be operated in its various order vibrational modes. However, the sensitivities of these modes can be different due to the vibration mode shape, as verified in Fig. 3, in which the sensitivity (slope rate of the linear fitting) at $801 \mathrm{kHz}$ is about 4 times higher than the higher frequency width-flexural or torsional modes. Furthermore, the normalized frequency shifts of different length flexural modes due to volatile absorption were identical, as shown in Fig. 4 with dashed and solid lines for each device. This measurement of equal normalized frequency shift for flexural modes for stress and mass accumulation is in line with theoretical predictions [6]. The higher sensitivity of the length-flexural modes can be explained by the frequency effect of the swelling-induced axial stress that accumulates most effectively in the longitudinal axis due to clamped-clamped boundary condition. 


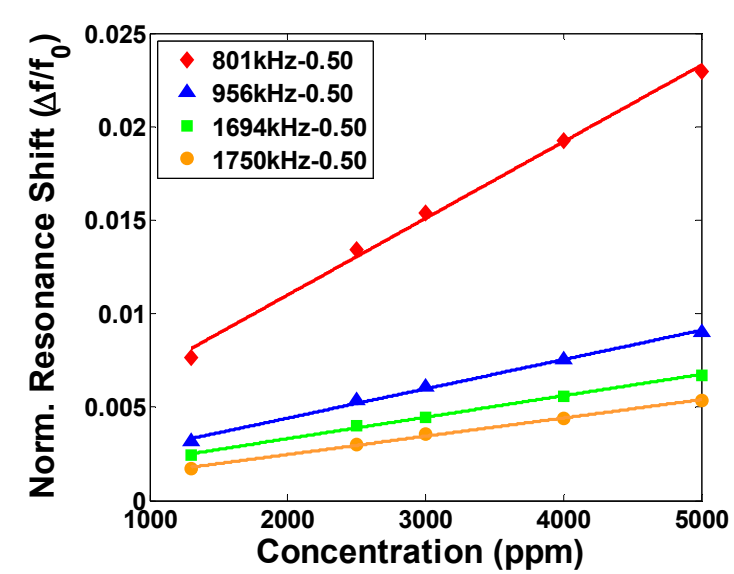

Fig.3 Normalized modal frequency shift as a function of the concentration levels of ethanol for different resonance modes, demonstrating the linearity of sensor response and higher sensitivity at lower length flexural modes.

\section{Size of piezoelectric transducer}

Among the parameters of interest for device performance is the piezoelectric transducer size, as this can be varied to optimize for electromechanical transduction efficiency. However, it is important to note that the size of the piezoelectric patch also plays an important role in the sensitivity, due to the thickness the patch adds to the structure, essentially stiffening the microbridge resonator and hence decreasing its dependence on the structural changes in the polymer layer.

Four resonators of identical geometry and matching layer thickness of PMMA, but different piezoelectric patch coverage $(0.1 \mathrm{~L}, 0.15 \mathrm{~L}$, $0.25 L$, and $0.5 L)$, where $L$ is the beam length, were tested at length-flexural modes. Their time trace curves of the relative frequency shift upon exposure to different concentration levels of ethanol are shown in Fig. 4. The extracted sensitivities after linearly fitting the amount of frequency shift against the concentration level can be seen in Fig. 5, in which the sensitivity dramatically decreases from $13 \times 10^{-6} / \mathrm{ppm}$ to $6 \times 10^{-6} / \mathrm{ppm}$ when the ratio increases from $10 \%$ to $25 \%$. The reduced sensitivity can be explained by the decrease in the ratio of the beam length $(L)$ over the beam thickness $(h)$. The resonators are $500 \mathrm{~nm}$ thick silicon nitride, and the transducer patch is approximately $600 \mathrm{~nm}$ thick stack of Pt-AIN-Pt. As such, as the piezoelectric patch length is increased, the effective beam thickness increased. The polymer thickness however is kept constant; therefore the relative sensitivity of the total composite structure to the accumulated change in the stress and the mass due to polymer swelling is decreased.

\section{Polymer thickness}

The uptake of volatile compound in an environment is directly determined by the partition coefficient of the vapor in the specific polymer, the concentration and the volume of the polymer [7]. For a fixed concentration and polymer-vapor pair, the mass uptake of volatile scales with the polymer thickness. Furthermore, for a fixed amount of layer stress, the relative modal frequency shift of beam resonator depends on the thickness of the stressed layer to the total thickness of the beam. As such, the overall sensitivity of the doubly clamped beam resonators, which depends on the combination of the accumulated change in stress and mass, is expected to be scale positively with polymer thickness.

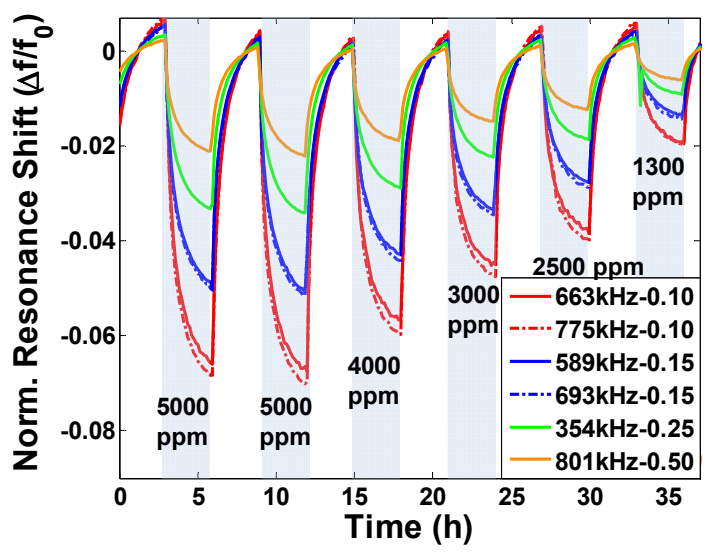

Fig. 4 Time response of four sensors, of identical length $L=750 \mu \mathrm{m}$, with different piezoelectric patch lengths $(0.1 \mathrm{~L}, 0.15 \mathrm{~L}, 0.25 \mathrm{~L}$ and $0.5 \mathrm{~L}$ as indicated in the legend) to various concentrations of ethanol exposure (blue regions) in dry nitrogen flow (white regions).

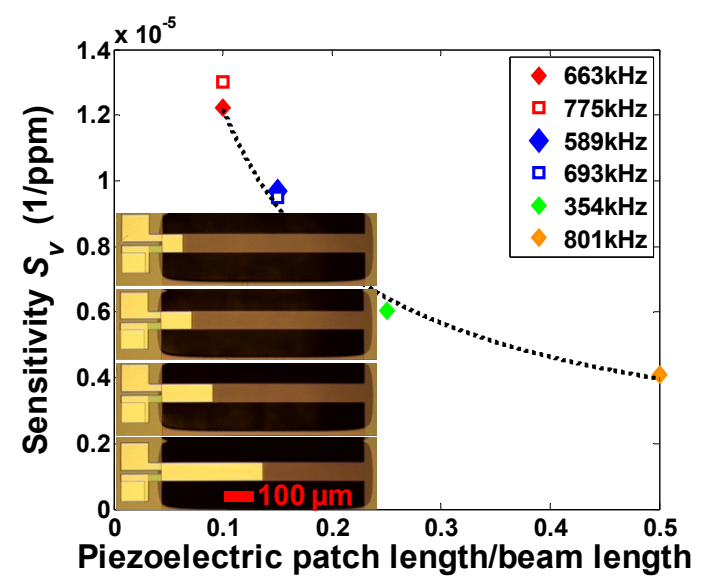

Fig. 5 Extracted sensitivities of identical polymer coating resonator sensors, operated at their length flexural modes, with varying piezoelectric transducer length, as shown in the inset. 
Indeed, the benefit of increasing polymer layer thickness is experimentally verified, as plotted in Fig. 6, where 4 resonators with identical geometry design, but different PMMA thicknesses are measured. The digital number $(1,3,5$ and 9$)$ in the figure is the layer number of printed PMMA. The sensitivity is around $5 \times 10^{-6} / \mathrm{ppm}$ with one layer $(70 \mathrm{~nm})$ of PMMA coating, and it increases up to $20 \times 10^{-6} / \mathrm{ppm}$ with 9 layers $(\sim 630 \mathrm{~nm})$ of PMMA coating.

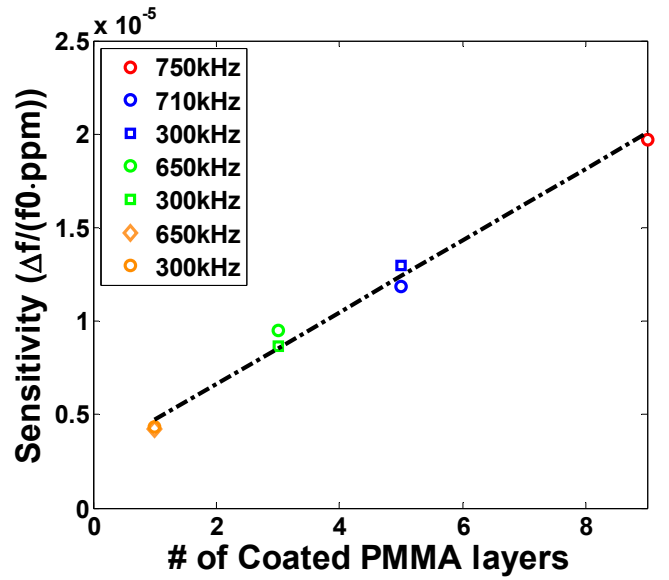

Fig. 6 Linear dependence of the resonator sensitivity to ethanol on the coated PMMA layer thickness for different lower order length flexural modes. Each layer of PMMA coating is estimated to be approximately $70 \mathrm{~nm}$ thick.

\section{Conclusion and discussion}

In this study, we have investigated several governing factors on the sensitivity of micromechanical beam resonators. Foremost, there exists a clear sensitivity advantage in employing lower frequency length-flexural resonance mode instead of higher-order width bending modes. However, it is clear that the order of the length flexural mode employed is influencing the sensitivity. Furthermore, it was determined that for a given thicknes of sensing layer, sensitivity is strongly dependent on the effective thickness of the beam. Finally, it is demonstrated in this study that sensitivity can be enhanced by increasing the polymer thickness.

This study clearly highlights the existance of several important trade-offs between the resonator sensitivity and the other sensor performance parameters. For example, increasing the layer thickness of polymer will consequently increases the sensor response time, and reducing the piezoelectric patch size will decrease the electromechanical coupling efficiency, resulting in weaker signals and requiring a higher amplification of the read-out circuit. As such, there exists a need to clearly define figure of merit for not only the resonant device alone but also taking into consideration the effect of the absorbent polymer and the accompanying read-out circuit specifications.

\section{References}

[1] D. Diamond, S. Coyle, S. Scarmagnani and J. Hayes, Chem. Rev. 108, 652-679 (2008)

[2] D. M. Karabacak, S. H. Brongersma, M. CregoCalama, Lab on a Chip, 10, 1976-1982 (2010)

[3] D. R. Southworth, L. M. Bellan and Y. Linzon, H. G. Craighead and J. M. Parpia, Applied Physics Letters, 96, 163503 (2010).

[4] M. K. Baller, H. P. Lang, J. Fritz, C. Gerber, J. K. Gimzewski, and U. Drechsler, H. Rothuizen, M. Despont, P. Vettiger, F. M. Battiston, J. P. Ramseyer, P. Fornaro, E. Meyer and H. J. Guntherodt, Ultramicroscopy, 82, 1-9(2000)

[5] K. Misiakos, I. Raptis, A. Gerardino, H. Contopanagos and M. Kitsara, Lab Chip, 9, 12611266(2009)

[6] M. V. Salapaka, H. S. Bergh, J. Lai, A. Majumdar, and E. McFarland, Journal of Applied Physics, 81, 2480-2487 (1997).

[7] J. W. Grate, A. Snow, D. S. Ballantine, H. Wohltjen, M. H. Abraham, R. A. McGill and P. Sasson, Analytical Chemistry, 60, 869-875 (1988) 